Göran Hammarström

\title{
Comment on Esa Itkonen's comment on Klaas Willems’ paper
}

I am puzzled to read that Itkonen just says that there are limits to linguistic intuition. He would probably agree with me if I say that a speaker is able to say that a word or a construction is correct, incorrect or of doubtful correctness. The speaker can also say that he has never heard an expression. If one investigates the intuition of several speakers, one will find that they agree on some points and disagree on other points. However, I do not think that this fact should be called a limit to intuition.

The fact that a speaker does not know all possible facts of his language is a special well known case which I think is of little interest. 